TAIWANESE JOURNAL OF MATHEMATICS

Vol. 4, No. 4, pp. 621-625, December 2000

\title{
NONEXISTENCE OF MONOTONIC SOLUTIONS OF SOME THIRD-ORDER ODE RELEVANT TO THE KURAMOTO-SIVASHINSKY EQUATION
}

\author{
Naoyuki Ishimura and MasaAki Nakamura
}

\begin{abstract}
We study the third-order ordinary differential equations (ODEs) which are relevant to the steady state of the Kuramoto-Sivashinsky equation, and/or to a model of dendritic growth of needle crystals. We show that there is no monotonic solution for certain range of parameter.
\end{abstract}

\section{INTRODUCTION}

We deal with the third-order ordinary differential equations (ODEs) of the form

$$
\lambda y^{\prime \prime \prime}+y^{\prime}=f(y), \quad y=y(x) \quad \text { for } x \in \mathbb{R},
$$

where $\lambda>0$ is a parameter. $f$ is a smooth function in a neighborhood of the interval $[-\alpha, \alpha]$, which is assumed to satisfy

(H1) $f( \pm \alpha)=0, f>0$ on $(-\alpha, \alpha), f^{\prime}( \pm \alpha) \neq 0$;

(H2) there exists a positive constant $a$ such that

$$
-2 f^{\prime \prime} f+\left(f^{\prime}\right)^{2} \geq a \quad \text { on }(-\alpha, \alpha) .
$$

Typical examples of $f$ include

$$
f(y)=1-y^{2} \quad \text { for which } \alpha=1 \text { and } a=4 ;
$$

${ }^{0}$ Communicated by H. Smith.

Received May 21, 1999.

2000 Mathematics Subject Classification: 34B15, 35Q35, 34C11.

Key words and phrases: Kuramoto-Sivashinsky equation, monotonic solution. 


$$
f(y)=\cos y \quad \text { for which } \alpha=\frac{\pi}{2} \text { and } a=1 .
$$

As is easily seen, the nonlinearity (2) is derived from the KuramotoSivashinsky equation $[9,10,14]$ :

$$
u_{t}+u_{x x x x}+u_{x x}+\frac{1}{2} u_{x}^{2}=0, \quad u=u(x, t) \quad \text { for } x \in \mathbb{R}, t>0 .
$$

If we postulate $u(x, t)=-2 \lambda t+v(x)$, which is numerically suggested by $[13,12]$, and put $y(x)=2^{-1} \lambda^{-1 / 2} v^{\prime}\left(\lambda^{-1 / 2} x\right)$, then we find (1) with (2); the solution in this case is usually referred to as steady state solutions of the Kuramoto-Sivashinsky equation [16]. The nonlinearity (3), on the other hand, is proposed as a simple model for two-dimensional dendritic growth of needle crysals in a supercooled liquid $[11,5]$.

For both (2) and (3), we have special interest in investigating whether there exists a solution $y$ satisfying $y^{\prime}(x)>0$ for all $x \in \mathbb{R}$ and $y(x) \rightarrow \pm \alpha$ as $x \rightarrow \pm \infty$, respectively.

Indeed, in a seminal work [8], it is observed that the odd monotonic solution of (1) with (3) verifying $y(0)=0$ and $y( \pm \infty)= \pm \pi / 2$ may have a discontinuity of $y^{\prime \prime}(0)$; to be precise, $y^{\prime \prime}(0) \neq 0$, and hence $y(x)$ cannot be odd. We remark that if $y(x)$ fulfills $(1),(3)$, then so does $-y(-x)$. Amick and McLeod [1] provide a mathematically rigorous demonstration on the nonexistence of monotonic solution of (1), (3); the method of [1] involves the complex variable and the theory of analytic functions. See also the foregone partial results by Troy [17].

Similar phenomena take place in the nonlinearity (2). Inspired somewhat by [8], Grimshaw and Hooper [4] indicate the nonexistence of monotonic solution with $y( \pm \infty)= \pm 1$ for (1), (2). Troy [17] gave an exact treatment if $\lambda=1 / 2$; he discusses the initial value problem $y(0)=0, y^{\prime}(0)=\beta>0$, $y^{\prime \prime}(0)=0$, and traces the trajectory in detail regarding $\beta$ as a parameter. Later, the complete nonexistence result for all $\lambda>0$ is established in [7]. The strategy for proof in [7] follows the line in [1] with refinement and necessary modifications. We hasten to remark that Toland [15] already showed the nonexistence property for (1), (2) if $\lambda \geq 2 / 9$. Toland employs elementary calculus only but very ingeniously.

In this note, motivated by the above examples, we pursue the nonexistence nature for general $f$; we exhibit rather applicable techniques to derive the desired consequence. Our main assertion reads as follows.

Theorem. Suppose $f$ fulfills the hypotheses (H1) and (H2). Then there is no solution of (1) which satisfies

$$
y^{\prime}(x)>0 \text { for all } x \in \mathbb{R} \text { and } y(x) \rightarrow \pm \alpha \text { as } x \rightarrow \pm \infty,
$$


if $\lambda \geq 32 /(27 a)$.

Note that our achievement is imperfect especially concerning the above examples (2) and (3) in the sense that it does not restore the nonexistence results for all $\lambda>0$. Nevertheless we wish to emphasize that the method is simple and universal enough to be applied to general $f$.

We conclude this introduction by a comment on researches in (1) from the viewpoint of the ODE community. It is evident that much attention has been paid on the analysis of (1) (we refer for instance to [3]); however, the concerns are laid exclusively on the case that $f$ is odd, even if the nonlinear functions are handled. Moreover, the connection with the mathematical physics does not seem to be taken into consideration.

\section{Proof of Theorem}

We proceed along the way in [6]. For the sake of contradiction, suppose that there exists a solution $y$ of (1) which verifies (4).

Since $y^{\prime}$ is positive definite, we use $y$ as an independent variable. This idea is introduced by [15] and independently by [2]. Let $x(y)$ denote the inverse function of $y(x)$ defined on $-\alpha \leq y \leq \alpha$. We put

$$
t=y \quad \text { and } \quad v(t)=\left\{y^{\prime}(x(t))\right\}^{2}
$$

and compute

$$
\begin{gathered}
v^{\prime}(t)=2 y^{\prime}(x(t)) x^{\prime}(t) y^{\prime \prime}(x(t))=2 y^{\prime \prime}(x(t)), \\
\lambda v^{\prime \prime}(t)=2 \lambda y^{\prime \prime \prime}(x(t)) x^{\prime}(t)=2 \frac{f(t)}{\sqrt{v(t)}}-2 .
\end{gathered}
$$

The linearization of (1) at the equilibrium point $y= \pm \alpha$ is

$$
\lambda \varphi^{\prime \prime \prime}+\varphi^{\prime}-y^{\prime}( \pm \alpha) \varphi=0,
$$

and the corresponding characteristic polynomial is

$$
\lambda z^{3}+z-f^{\prime}( \pm \alpha)=0 .
$$

Since $f^{\prime}(\alpha)<0$ and $f^{\prime}(-\alpha)>0$, we infer that $v^{\prime}( \pm \alpha)=0$ taking account of the stable manifold theorem; the real part of one root of the characteristic polynomial is negative for the case $y=\alpha$ and positive for $y=-\alpha$. 
To summarize, as the result of transformation (5), we have deduced the next overdetermined problem from (1), (4).

$$
\begin{aligned}
& \lambda v^{\prime \prime}(t)=2 \frac{f(t)}{\sqrt{v(t)}}-2, \quad v(t)>0 \quad \text { for }-\alpha<t<\alpha, \\
& v( \pm \alpha)=v^{\prime}( \pm \alpha)=0 .
\end{aligned}
$$

Next we prepare a comparison function

$$
w(t)=M \sqrt{f(t)},
$$

where $M>0$ will be determined later. We perform the calculation

$$
\begin{aligned}
w^{\prime}(t) & =\frac{M}{2} \frac{f^{\prime}(t)}{\sqrt{f(t)}} \\
\lambda w^{\prime \prime}(t) & -2 \frac{f(t)}{\sqrt{w(t)}}+2 \\
& =-\frac{1}{f^{3 / 2}}\left\{\frac{\lambda M}{4}\left(-2 f^{\prime \prime} f+\left(f^{\prime}\right)^{2}\right)+\frac{2 f^{9 / 4}}{\sqrt{M}}-2 f^{3 / 2}\right\} \\
& \leq-\frac{1}{f^{3 / 2}}\left(\frac{\lambda M}{4} a+\frac{2 f^{9 / 4}}{\sqrt{M}}-2 f^{3 / 2}\right) .
\end{aligned}
$$

Now the function $g(X):=4^{-1} \lambda M a+2 M^{-1 / 2} X^{9 / 4}-2 X^{3 / 2}$ takes the minimum value $g=\left(4^{-1} \lambda a-8 / 27\right) M$ at $X^{3 / 4}=2 \sqrt{M} / 3 ; w(t)$ becomes a supersolution if $\lambda \geq 32 /(27 a)$, irrespective of the value of $M$. In view of $w( \pm \alpha)=0$ and $w^{\prime}( \pm \alpha)=\mp \infty$, we can make $w(t)$ touch $v(t)$ from above at the interior, by letting $M$ large first and then decreasing it. The interior touching principle prohibits such a situation. This absurdity leads us to the required conclusion.

\section{ACKNOWLEDGEMENTS}

This work is partially supported by Grants-in-Aids for Scientific Research (Nos. 10354110, 10555023), Japan Ministry of Education, Science, Sports and Culture.

\section{REFERENCES}

1. C. Amick and J. B. McLeod, A singular perturbation problem in needle crystals, Arch. Rational Mech. Anal. 109 (1990), 139-171. 
2. F. Bernis, L. A. Peletier and S. M. Williams, Source type solutions of a fourth order nonlinear degenerate parabolic equation, Nonlinear Anal. 18 (1992), $217-234$.

3. M. Greguš, Third Order Linear Differential Equations, D. Reidel Publishing Company, Dordrecht, 1987.

4. R. Grimshaw and A. P. Hooper, The non-existence of a certain class of travelling wave solutions of the Kuramoto-Sivashinsky equation, Phys. D 50 (1991), 231238.

5. J. M. Hammersley and G. Mazzarino, A differential equation connected with the dendritic growth of crystals, IMA J. Appl. Math. 42 (1989), 43-75.

6. N. Ishimura and M. A. Nakamura, A note on the nonexistence of monotonic steady solutions of the Kuramoto-Sivashinsky equation, preprint.

7. J. Jones, W. C. Troy and A. D. MacGillivary, Steady solutions of the KuramotoSivashinsky equation for small wave speed, J. Differential Equations 96 (1992), $28-55$.

8. M. D. Kruskal and H. Segur, Asymptotics beyond all orders in a model of crystal growth, Stud. Appl. Math. 85 (1991), 129-181.

9. Y. Kuramoto and T. Tsuzuki, Persistent propagation of concentration waves in dissipative media far from thermal equilibrium, Progr. Theoret. Phys. 55 (1976), 356-369.

10. Y. Kuramoto and T. Yamada, Turbulent state in chemical reaction, Progr. Theoret. Phys. 56 (1976), 679.

11. J. S. Langer, Existence of needle crystals in local models of solidification, Phys. Rev. A 33 (1986), 435-441.

12. D. Michelson, Steady solutions of the Kuramoto-Sivashinsky equation, Phys. D 19 (1986), 89-111.

13. D. M. Michelson and G. I. Sivashinsky, Nonlinear analysis of hydrodynamic instability in laminar flames II. Numerical experiments, Acta Astronautica 4 (1977), 1207-1221.

14. G. I. Sivashinsky, Nonlinear analysis of hydrodynamic instability in laminar flames I. Derivation of basic equations, Acta Astronautica 4 (1977), 1177-1206.

15. J. F. Toland, Existence and uniqueness of heteroclinic orbits for the equation $\lambda u^{\prime \prime \prime}+u^{\prime}=f(u)$, Proc. Roy. Soc. Edinburgh Sect. A 109 (1988), 23-36.

16. W. C. Troy, The existence of steady solutions of the Kuramoto-Sivashinsky equation, J. Differential Equations 82 (1989), 269-313.

17. W. C. Troy, Nonexistence of monotonic solutions in a model of dendritic growth, Quart. Appl. Math. 48 (1990), 209-215. 
Naoyuki Ishimura

Department of Mathematics, Hitotsubashi University

Kunitachi, Tokyo 186-8601, Japan

MasaAki Nakamura

College of Science and Technology, Nihon University

Kanda-Surugadai, Tokyo 101-8308, Japan 